\title{
Arrêts sur image : fragments d'une identité-flux
}

\author{
Bertrand Gervais \\ Université du Québec à Montréal
}

Depuis 2009, l'artiste canadienne Suzy Lake a commencé une série d'autoportraits grandeur nature, intitulée Reduced Performing. Les images de cette série sont étrangement statiques. On y voit l'artiste suspendue dans les airs, impression accentuée au moment de l'accrochage en galerie. Les perspectives sont aplaties; le corps y paraît inerte, comme s'il s'agissait d'une dépouille. Tout semble artificiel : la prise de vue frontale écrase la masse du corps, aucun élément de décor ne transparaît, les mains sont déposées sur les cuisses ou sur le côté sans aucune grâce, les lunettes ne reposent pas de façon 
naturelle sur le nez, même les yeux manquent d'expressivité, rendus énigmatiques par un certain flou. Que regarde Suzy Lake? Regarde-t-elle même quelque chose? Sur «Breathing \# 5 », où l'artiste porte un chandail bleu rayé de noir, le haut du torse est l'objet d'une étonnante saturation. Le tissu perd de sa netteté, du vert apparaît, un bleu plus intense. Les contours d'un pentagone irrégulier sont dessinés. On finit par saisir que cette distorsion visuelle est la conséquence des respirations prises par l'artiste au moment de la prise de vue.

C'est que les clichés qui composent cette performance réduite, voire minimale, ont été réalisés à l'aide d'un numériseur grand format. Les photos n'ont pas été prises avec un appareil photo, mais avec un scanner, dont le processus de captation par balayage est lent et fastidieux, ne se calculant pas en centièmes ou en millièmes de seconde, mais en minutes, en véritables minutes. L'appareil utilisé par Lake met sept minutes à réaliser chacune des images. Comme le signale le catalogue de l'exposition de 2013 du Mois de la photo à Montréal, où ont été exposées ces photos, "[d]urant cette "exposition" prolongée, Lake respirait, clignait des yeux et pleurait parfois. [...] Il en résulte une image d'une étrange immuabilité qui se situe au croisement de l'image stylisée du catalogue et de la figure sainte descendue du ciel. » (2013, p. 84)

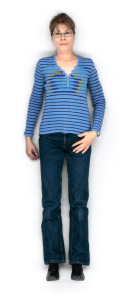

Suzie Lake, « Reduced Performing : Breathing \# 5 », 2009 
Le corps de Lake est ainsi capté par une technologie qui a été détournée de sa fonction initiale. L'efficacité des appareils photos numériques permet de capter des mouvements, même les plus furtifs. Avec un scanner fait pour reproduire des formes immobiles, le mouvement, tel que le clignement des yeux ou la respiration, qui implique l'expansion et la compression de la cage thoracique, apparaît sur l'image comme des zones floues. C'est du bruit. Le scanner n'est pas fait pour capter la vie, mais pour reproduire une image le plus efficacement possible. Les manifestations de la vie y sont excédentaires; elles échappent à la logique immobilisante du balayage du numériseur. En fait, l'objet même de la performance de l'artiste au cœur de la série est constitué de ces mouvements involontaires et habituellement négligés, parce que tenus pour acquis, battements de paupières, sécrétion de larmes, mouvements respiratoires, réflexes qui sont l'occasion, par le biais du dispositif utilisé, d'un fascinant procédé de défamiliarisation. Suzy Lake révèle elle-même l'objectif poursuivi par son projet : "Comment [...] réduire la dimension narrative de l'activité photographique tout en conservant une lecture du corps qui se révèle dans le temps?» (2013, p. 84) Reduced Performing le réalise en étirant le temps d'exposition, par la voie d'un dispositif détourné, qui est un véritable appel à l'histoire de la photographie, quand au XIXe siècle les temps d'exposition se calculaient justement en dizaine de minutes.

Le corps numérisé par Lake est un corps sans artifices, ou si peu, un corps devenu pure surface, sans aucune intériorité, un corps immobilisé pour les besoins de la cause, rigidifié comme s'il était atteint de rigor mortis, étendu de tout son long sur une surface blanche; mais c'est en même temps un corps reproductible à l'infini dans sa surface même, parce que, fait 
maintenant de pixels, il peut être intégré à un réseau, au cyberespace qui en démultipliera la présence et en diffusera les images.

C'est un corps de pixels, un corps-image, conçu pour être manipulé, mais en même temps, c'est un corps qui a choisi de faire acte de résistance et qui doit se refermer sur lui-même afin d'accomplir une tâche, celle d'être immobile, le plus immobile possible. C'est donc un corps dont on s'échappe. À quoi Suzy Lake pense-t-elle pendant ces sept minutes? Est-elle attentive à ce qui se passe ou cherche-t-elle plutôt à s'extraire par la pensée de sa situation? Est-elle en train de muser, son esprit à mille lieues de son corps? Le numériseur finit par ressembler à un sarcophage qui ne réussit à retenir que le superficiel, la forme du corps, son volume, ses traits, le reste étant perdu, puisqu'impossible à capter.

Le corps numérisé est un corps-surface, un corps sans intériorité, sans intimité. C'est un corps soumis aux formes de l'extimité, où l'identité est déterminée par des flux (ici un flux de lumière) plutôt que par une permanence.

\section{Une culture de l'image}

L'expérience de Suzy Lake dans Reduced Performing permet d'appréhender le spectre de la diversité des formes d'inscription numérique du sujet contemporain. Celui-ci a non seulement le choix du dispositif, du plus simple au plus complexe, mais encore du degré d'intensité de son inscription, de la plus superficielle à la plus complète, allant des versions de soi, numérisées de quelque façon que ce soit, aux personnages et aux corps virtuels des arts médiatiques, qui sont souvent de 
pures créations numériques. Au corps étrangement statique de Reduced Performing répond, par exemple, la figure fantomatique de Encoded Presence, le projet de Magruder de 2005 (Gervais et Desjardins, 2009, p. 9-23). L'image y est fortement, pour ne pas dire exagérément pixellisée; le corps dessiné à grands traits se déplace tel un spectre dans l'espace rectangulaire de la fenêtre compacte d'un téléphone cellulaire. Impossible de reconnaître qui que ce soit. L'objectif n'est pas d'immobiliser le corps, mais d'en signifier la présence, une présence précaire, furtive, mais tenace malgré tout. Si le mouvement dans Reduced Performing produit du flou et paraît excédentaire, il s'impose comme le principe même de Encoded Presence, qui n'offre rien d'autre à voir qu'une animation rudimentaire.

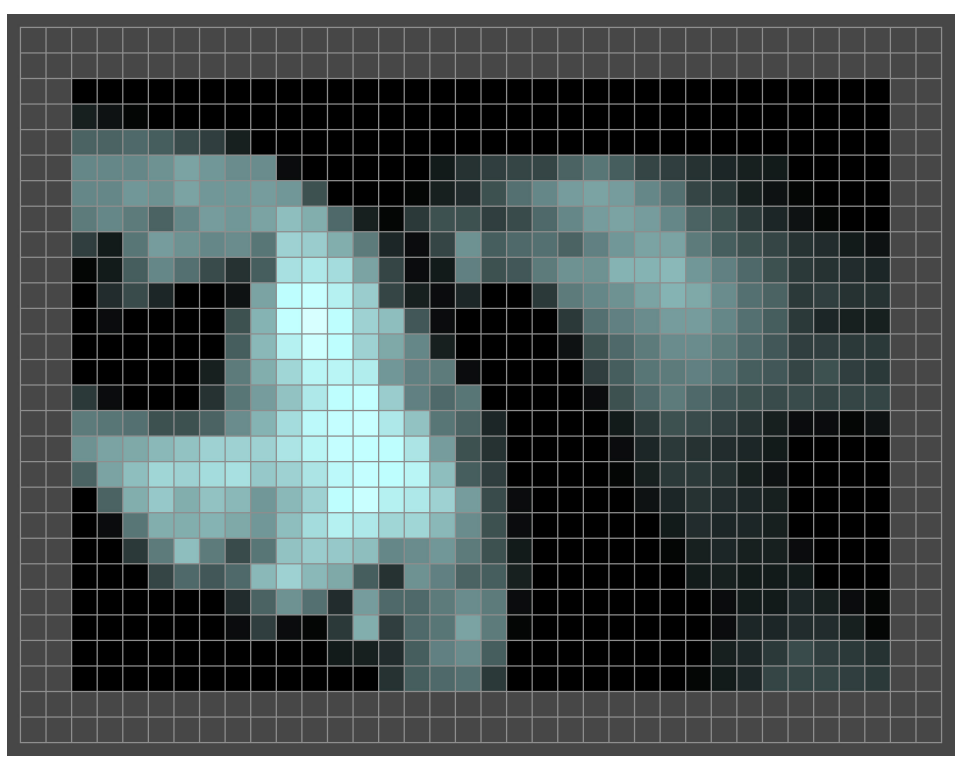

Michael Takeo Magruder, Encoded Presence, 2005 (capture d'écran) 
Penser le sujet digital, ou plus précisément l'expression de la subjectivité en contexte numérique, c'est penser les formes de l'imaginaire contemporain. Un imaginaire marqué par une culture de l'écran de plus en plus prépondérante. Pour décrire cet ordre, on peut, à la suite de Christine Buci-Glucksmann, parler d'une folie du voir. Comme elle le souligne, «de l'omnivoyance baroque à celle du virtuel, de ses panoptismes et de ses interfaces, on a assisté à la naissance et au développement d'une nouvelle folie $d u$ voir à l'échelle mondiale » (2002, p. 227). Cette folie du voir étend son emprise sur de nombreuses sphères de nos activités sémiotiques et elle s'inscrit comme trait saillant d'une culture de l'écran, où la dimension iconique des interfaces prescrit plus que jamais son ordre symbolique singulier. Elle joue explicitement sur une contribution des images non seulement comme mode de représentation, mais comme modalités identitaires et processus de connaissance. Ce sont les deux aspects, identitaire et cognitif, sur lesquels je veux surtout m'arrêter ici. Et je le ferai en fonction de la place qu'occupe l'image, par la voie de la photographie, et l'écran, en tant qu'interface, dans nos habitudes et nos façons de nous définir.

Un premier exemple simple de cette folie du voir, ancrée en pleine culture de l'écran, c'est ce qu'on peut appeler le Dictionnaire visuel Google (DVG). Le DVG, ce sont les pages de résultats "images » du moteur de recherche Google. Il semble bien qu'à notre époque, tout soit visible, tout se donne à voir, jusqu'à l'invisible et l'impalpable. C'est la grande leçon des moteurs de recherche, et surtout du développement des métadonnées permettant d'indexer les photos et images téléversées sur des sites de partage ou des blogues. La page de résultats « images » du moteur Google offre ainsi des catalogues 
étonnants d'images pour des interrogations qui devraient normalement ne rien donner. Strictement ne rien donner. Prenons le mot «invisible» (par définition, «qui n'est pas visible, qui échappe à la vue»). Entrons-le dans le DVG et, presto, on obtient vingt-cinq pages de résultats. Vingt-cinq pages de photos qui vous montrent l'invisible dans toute sa splendeur. L'homme invisible. La main invisible qui se fait sentir sur un corps. La voiture en partie effacée grâce à Photoshop. Des chats dans des poses étonnantes (pourquoi estce que les chats servent mieux que tout autre être vivant à figurer l'invisible? La question laisse perplexe...).

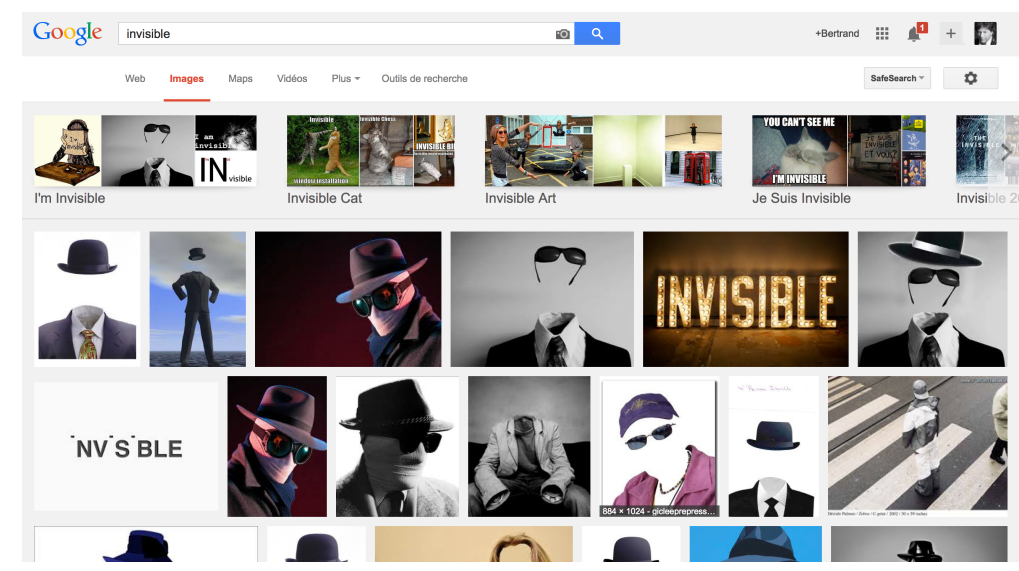

Capture d'écran des résultats « images » du moteur de recherche Google

De tels résultats sont, on le comprend aisément, paradoxaux. L'invisible, c'est ce qui ne se donne pas à voir. Pourtant, les résultats nous en offrent de multiples versions, toutes plus insignifiantes les unes que les autres. Entrez les mots «vide 
absolu», «rien», « intangible», «irréel», « imperceptible», « indécelable », « deus ex machina », tous permettent d'obtenir des résultats. Des pages et des pages de photos de choses qui ne devraient pas pouvoir être vues. C'est dire que plus rien n'échappe au visible. Nous sommes, par le biais de la culture de l'écran qui se généralise, dans un régime de la visibilité absolue, et parfois même de l'absurdité absolue.

Tout est image. Il n'y a plus de mystère. Les symboles les plus ineffables s'offrent au regard. En même temps, tout est dénaturé. Car les images récupérées par le DVG ne portent pas sur les notions elles-mêmes, sauf exception, mais sur des marques de commerce. Ce sont des attributs de produits. Synec.doc, une firme spécialisée dans les sites web (un vrai cas de synecdoque...). Deus ex machina, un fabriquant de motos. Imperceptible, les condoms Skyn. Invisible, la lingerie Passionata, etc. Même les termes les plus abstraits génèrent leur poids en images concrètes. L'effet est pour le moins insolite, comme si notre époque n'était plus capable de penser abstraitement; comme si le cyberespace, pourtant abstrait par définition, rendait tout concret, palpable ou visible. Et on finit par s'interroger: quelle proportion du dictionnaire est représentée en images? Existe-t-il encore des mots qui ne sont pas associés à des images? Qui ne donnent aucun résultat dans le DVG? Même le rien et le vide absolu donnent des résultats visuels. La conséquence est simple: plus rien n'échappe au visible. 


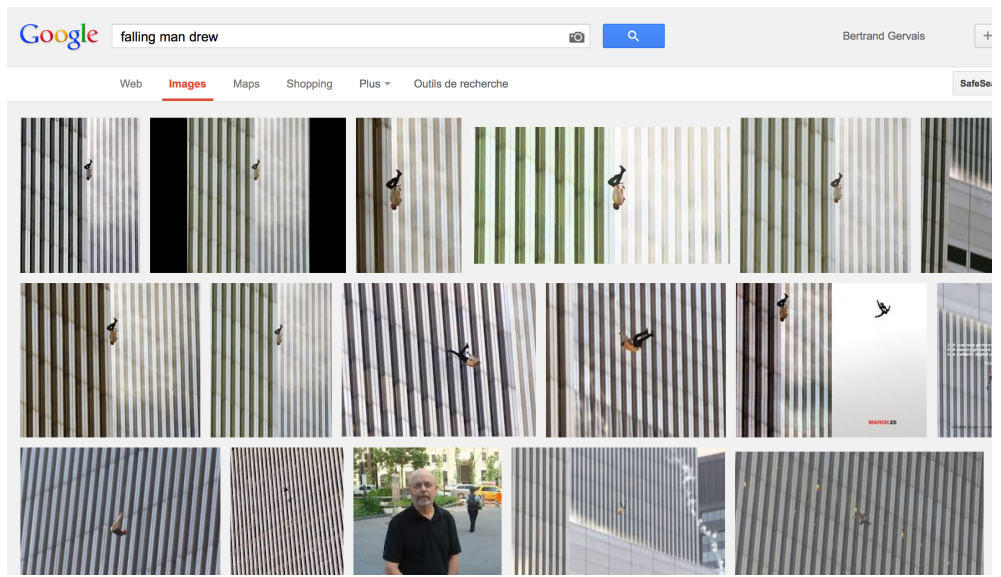

Résultats images de « The Falling Man » : la multiplication des images

Un autre effet notable du Dictionnaire visuel Google est le principe de généralisation et de multiplication des images. Une image singulière se voit multipliée presque à l'infini dès l'instant où elle suscite l'intérêt. L'exemple par excellence en est «L'homme qui tombe» / «The Falling Man», la célèbre photographie du photojournaliste de l'Associated Press, Richard Drew, prise au moment des attentats du 11 septembre 2001. Prise à $9 \mathrm{~h} 41 \mathrm{~min} 15 \mathrm{sec}$ le 11 septembre, la photographie montre un homme en chute libre, la tête en bas, se découper du fond uniforme de l'image constitué par la paroi des deux gratte-ciel. Cette figure est devenue avec les années un des motifs les plus importants des fictions du 11 septembre. Il est évidemment facile de trouver cette photographie sur Internet. Il suffit d'entrer le titre de la photo, que l'on doit à un journaliste du magazine Esquire, sur un moteur de recherche. Mais les résultats de la requête donnent lieu à un déferlement d'hommes qui tombent. La photo de Drew et de nombreux 
autres clichés des tours en flammes et de jumpers défilent à l'écran, entrecoupés d'images indirectement liées aux attentats, les unes associées au générique de la série américaine Mad Men, les autres à des figures sculptées ou peintes d'hommes défenestrés ou simplement en chute libre, tel Icare.

La photo unique de DExtimitérew, celle d'un homme immobilisé en plein vol par un procédé photographique, se trouve reproduite presqu'à l'infini sur le DVG. L'image est dématérialisée, réduite à la taille d'une vignette, banalisée à l'extrême, mais sa réitération sur la page de résultats du DVG lui donne un certain mouvement, comme si l'écran était le lieu d'un flip book d'un nouveau genre. À faire défiler le mur de photographies des Falling Man, on finit par identifier la page du DVG à la paroi d'un gratte-ciel contre laquelle l'homme se remet à glisser, dans un simulacre improvisé de la chute initiale. La multiplication participe à un étonnant processus de mythification, comme si, d'un événement ponctuel, la chute devenait la séquence rejouée ad nauseam d'un récit réduit à sa plus simple expression ${ }^{1}$.

\section{Extimité}

Le sujet digital est marqué par une folie du voir, une exacerbation du rôle de l'image dans toutes les sphères de l'agir humain. Les images ne font plus que nous accompagner, elles orientent notre regard et, en culture de l'écran, nous imposent leur ordre sémiotique et symbolique. Pour Buci-Glucksmann, la transition d'une culture du livre à une culture de l'écran impose

${ }^{1}$ Dans Gervais (2014), j'ai exploré de façon soutenue cette figure de l'homme qui tombe. 
aussi «le passage d'une culture des objets à une culture des flux, des écrans et des doubles » (2002, p. 230), une culture où l'identité se négocie comme une monnaie. Nous sommes de plus en plus la somme des images par lesquelles les autres nous perçoivent. C'est le fondement même de l'extimité et de l'identité-flux qu'elle détermine.

L'extimité, comme fondement des rapports identitaires, se développe de façon majeure en culture de l'écran. Ce néologisme a été développé en concept par Serge Tisseron. Le psychanalyste explique que l'intimité, en tant que gage de l'authenticité de l'expérience, n'est plus une donnée essentielle à la façon dont un sujet se définit. À la métaphore d'un noyau identitaire, cette vérité du sujet qui est, sauf exception, tenue à l'abri des regards indiscrets, succède celle d'une surface d'identification en constante négociation. Pour Tisseron, l'extimité représente «le mouvement qui pousse chacun à mettre en avant une partie de sa vie intime, autant physique que psychique » (2001, p. 52). Ce mouvement vers l'extimité a toujours existé, mais ce qui est nouveau, «c'est sa revendication et, plus encore, la reconnaissance des formes multiples qu'il prend» (p. 52). Toute technique s'accompagne "d'une posture psychique qu'elle induit et qui influe, à son tour, sur le contenu du message» (p.63). La définition d'une extimité permet d'exprimer les changements qu'Internet provoque sur le plan des mécanismes de projection et d'identification au cœur de cette posture. Le cyberespace lui fournit «un espace pratiquement infini pour se manifester» (Tisseron, 2006, p. 6) et elle le fait d'une façon prépondérante. 


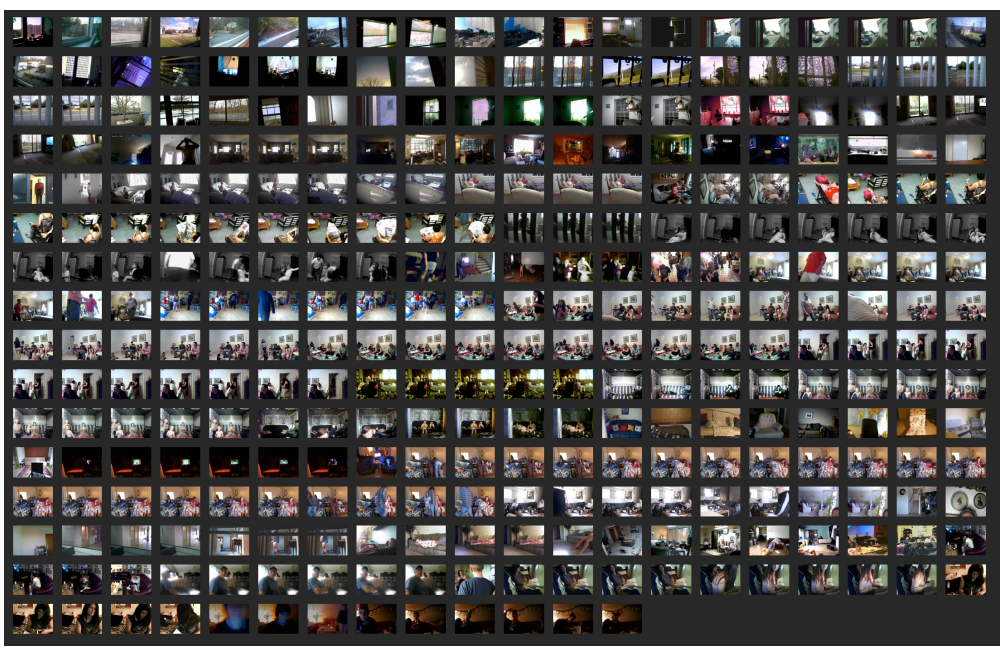

Capture d'écran du projet Everybody's Autobiography de Cheryl Sourkes

Dans Everybody's Autobiography, Cheryl Sourkes explore des sites d'hébergement de webcams afin d'y récupérer des images. Cela donne une mosaïque fascinante de scènes de vie, toutes plus banales les unes que les autres. Des hommes et des femmes se filment dans leur salon en train de regarder la télévision, de manger des croustilles, de lire, de jouer avec leurs enfants ou de dormir. Le projet nous transforme en voyeurs. En témoins d'un spectacle tout aussi insignifiant que futile. Pourquoi ces gens se filment-ils? Que gagnent-ils à ce que leur image soit diffusée? Quel œil bienveillant transformera leur quotidien en événement? Le projet de Sourkes, en offrant un échantillon d'images réunies en un grand tableau, constitue un véritable Panopticon, où un observateur distant et invisible peut à sa guise surveiller et contrôler un ensemble d'individus, chacun capté dans sa cellule à toute heure du jour ou de la nuit. Mais c'est un Panopticon sans possibilité d'action, un simulacre 
de dispositif de surveillance, puisque les images des webcams ont été enregistrées et que l'observateur n'a aucune possibilité d'intervention. Sourkes signale d'emblée le caractère évanescent des webcams, dont elle se sert de façon majeure dans ses œuvres et dispositifs :

Les photographies disparaissent presque aussitôt qu'elles apparaissent. Les images de ces caméras sont des résidus virtuels qui participent à une économie du rebut et de la décharge. Ce sont des portails vers des endroits inaccessibles. Réunies, elles constituent cependant un portrait de la culture contemporaine. (Sourkes, 2013; je traduis)

Un portrait à caractère ethnographique, nullement intentionnel. Un portrait qui parle aussi d'un irrépressible besoin de prendre place, de quelque façon que ce soit, dans le grand échange d'images que le réseau permet.

\section{Identité-flux}

Buci-Glucksmann parle de cette extimité en termes de Moi-toile, expression forgée à partir de la notion de Moi-peau de Didier Anzieu. Le Moi-toile se présente comme un feuilleté, constitué par «une multiplicité de plans d'inscriptions, qui s'entreexpriment et se surimpriment dans un aller et retour incessant » (2002, p. 260). Le Moi-toile est une identité-flux en constante renégociation : «[Il] introduit une véritable perte de territoire identitaire par des passages continus de frontières, de sensibles et d'arts. Car le corps élargi ouvre à un véritable "théâtre des interfaces", avec sa pluralité de regards et son intersensorialité. » (p. 262)

Quel que soit le terme utilisé, celui de Moi-toile, de Moiécran ou d'extimité, tous recouvrent une même réalité, celle 
d'une identité-flux, permettant de cerner les rapports identitaires établis dans le cyberespace ou, plus largement encore, établis en culture de l'écran, voire de l'écran relié, si par là on veut bien entendre l'écran d'un ordinateur ouvert sur un réseau de communication. Ce sont des identités qui font écran. L'identité-flux s'impose en fait comme une identité cybernétique, au sens d'une identité virtuelle établie en situation de communication, situation provisoire quoique stable, puisque déployée sur la base d'un réseau pérenne.

L'identité est le produit de la rencontre de multiples flux, que ce soit celui des événements quotidiens ou le flux de nos propres pensées qui nous constituent comme sujet. Évidemment, ce flux, dans le cyberespace, n'est plus une métaphore permettant de conceptualiser le mouvement et les processus en acte, il s'impose comme une réalité phénoménologique. De nombreux artistes web jouent avec cette identité-flux. Mark Amerika en parle d'ailleurs en termes de «digital flux persona » (2011, p. 12 et passim), qui apparaît de plus en plus comme un troisième terme venant complexifier l'opposition établie par Paul Ricœur entre identité-ipse et identité-idem. Au couple oppositionnel du propre (ipsé) et du semblable (même), répond l'identité-flux en continuelle renégociation. C'est une identité différentielle, en processus permanent d'ajustement.

Quelles sont les caractéristiques de cette identité-flux? La première est évidemment la prépondérance des dispositifs techniques, qui servent d'appui à cette identité. Les paramètres de l'identité-flux sont déterminés afin de répondre aux exigences du numérique et de la culture de l'écran. La deuxième est l'instabilité des marqueurs identitaires, qui permettent tous 
les jeux, à l'aide de pseudos et d'avatars, de constructions de personæ complexes, de manipulations de corps virtuels et de figures imaginaires. Ces jeux favorisent un brouillage de plus en plus important des limites de la fiction, processus qui est au cœur même de la culture de l'écran. En boutade, on pourrait dire que nous sommes des Zelig numériques. Dans son film éponyme de 1983, Woody Allen met en scène un véritable homme-caméléon qui change de corps au gré des rencontres, se transformant en rabbin, en Afro-Américain ou en Chinois, le temps d'une brève rencontre. L'identité-flux détermine, si on me permet le néologisme, une zeligation identitaire ou un caméléonisme numérique, qui nous permet d'adopter la figure que nous voulons, vraisemblablement sans conséquence physiologique notable.

Une troisième caractéristique est l'importance qu'y joue l'image, toutefois une image faite non pas pour être regardée mais manipulée. Et une image qui occupe le devant de la scène. C'est bien ce que les réseaux sociaux nous disent, de Facebook à Google+. Nous sommes dans une logique de l'anecdotique et du superficiel, mais, plus important encore, de l'immédiatement présent et du renouvelable à l'infini. Sur les réseaux, nous sommes la somme des images que nous avons mises ou qui ont été mises en ligne et où nous figurons. Nous existons virtuellement à la croisée des flux - flux urbain, flux des capitaux, flux informationnel d'un réseau accessible depuis un écran, flux interne de la pensée -, et ce sont ces flux qui déterminent l'identité que nous pouvons adopter. 


\section{L'ordre des images}

L'écran, surtout depuis la généralisation de l'ordinateur graphique, implique une interface fondée sur les relations iconiques, qui repose par conséquent sur l'image et ses particularités médiatiques et sémiotiques. La culture de l'écran entérine en fait une réalité de plus en plus prégnante, celle d'une image devenue processus de connaissance. Cette image, on la regarde et on la manipule, on s'en sert pour connaître et se reconnaître, et son régime sémiotique singulier impose sa logique associative et relationnelle.

Il convient de distinguer, comme le fait Tisseron, une image objet et un "objet-image ». Aborder l'image comme un objet, comme il le fait, permet de prendre en compte «l'ensemble des relations que nous entretenons avec elle. Ces relations engagent sa matière, son poids, son épaisseur, le jeu de son recto et de son verso, mais aussi la possibilité de la manipuler et de la transformer. » (1999, p. 109-110) Tisseron continue en disant :

Ce qui caractérise le règne de l'objet-image, c'est que la relation aux contenus des images ne peut plus être envisagée sans prendre en compte la relation physique et psychique que le sujet entretient avec son support. Cette approche implique de renoncer au paradigme habituellement retenu pour parler des images, celui du miroir. L'image n'est pas un miroir, ou plutôt, elle est un miroir que l'on transforme avec les mains. (p. 109-110)

L'image n'est plus faite pour être regardée, ou pour représenter, mais pour imposer un ordre ou entretenir une identité. Elle est un miroir qu'on transforme en le manipulant. Un miroir qui sert à générer des doubles, à reproduire des formes, en les multipliant. Le moment narcissique du reflet peut être non seulement étiré, soutenu dans une durée, mais devenir le lieu 
d'un travail, d'une série de manipulations qui viennent brouiller les frontières entre le réel et le fantasme, entre soi et la figure que l'identité-flux permet de mettre en circulation.

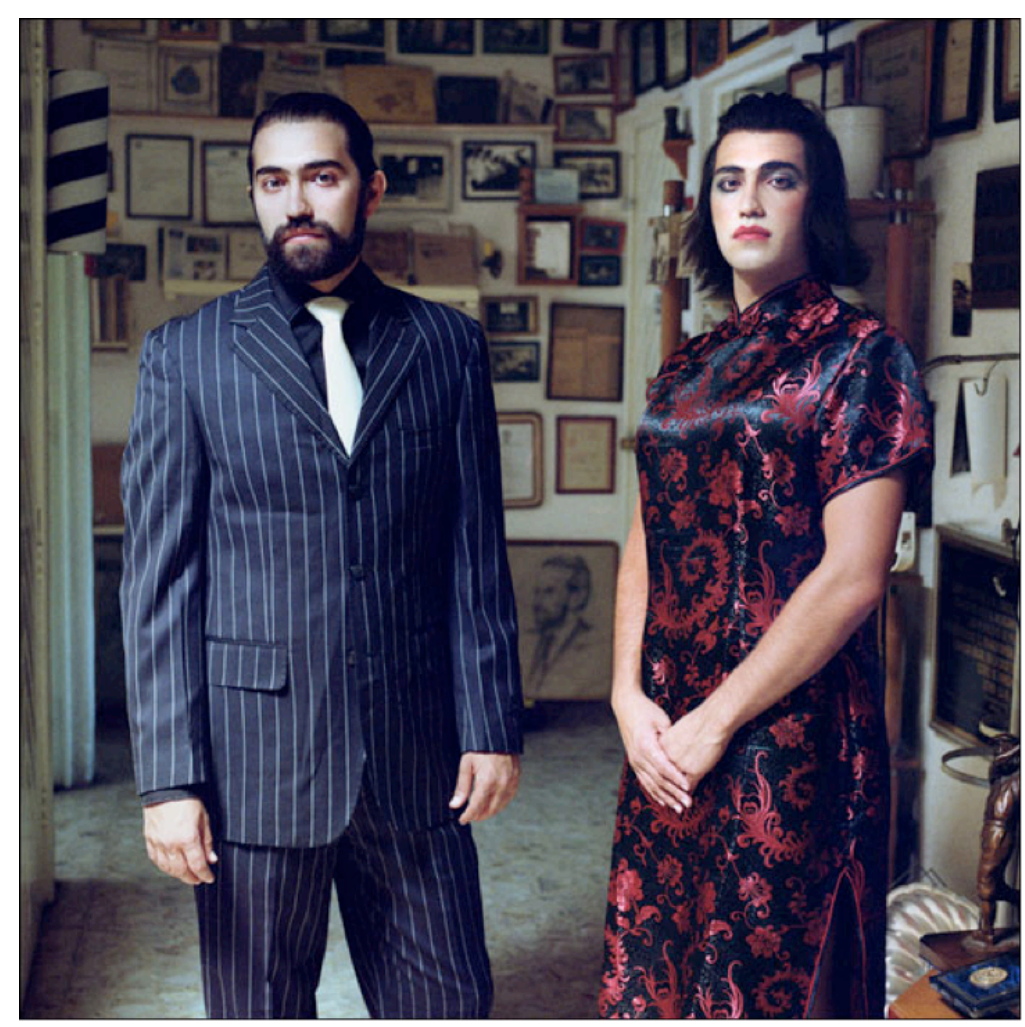

«Los Hermanos Miranda », photographie de Pierre Dalpé

Un photographe tel que Pierre Dalpé met ces possibilités techniques du numérique à contribution en créant des images d'une inquiétante étrangeté. Dans sa série intitulée Personæ, 
accessible en ligne sur son site web, mais disponible aussi sous forme de livre, il multiplie les photographies de jumeaux. Que ce soient les photos de Brandi et de Tammy, des frères Miranda, des sœurs Siddiqi ou encore des jumelles Forrest, chacune offre, dans une mise en scène d'une grande banalité, le spectacle de jumeaux, voire de triplets. Ces photos sont des constructions. Du moins pour la plupart. Les jumeaux et jumelles de la série Personæ sont des fabrications, le photographe ayant demandé à ses sujets de poser deux ou trois fois dans des versions différentes d'eux-mêmes. Le travail de composition de l'image se fait dans l'après-coup, à l'aide du numérique. Et le rendu recherché n'est pas la création de créatures virtuelles ou fantomatiques, mais bien plutôt de doubles tout ce qu'il y a de plus banals, captés dans des lieux ordinaires, à la manière de Diane Arbus, par exemple. On peut aisément, si on ne connaît pas le travail du photographe, prendre ses sujets pour de véritables jumeaux. Comme le signale Dayna McLeod, «Personæ respire l'authenticité en raison de sa perfection formelle et remet en question la pertinence culturelle de tout ce qui constitue des copies, qu'il s'agisse d'êtres humains, d'images numériques ou d'objets tangibles comme des photographies » (2010, p. 21). La frontière entre le vrai et le faux, du moins entre l'authentique et le fabriqué, est indiscernable. Ce n'est qu'à la répétition, en étant confronté à plus d'une photographie, qu'on parvient, comme spectateur, à avoir des soupçons sur le caractère fabriqué des clichés. Et, dès que nous comprenons que les sujets de Dalpé sont des doubles et que les photos sont le résultat d'un savant montage, pour ne pas dire d'un trucage, "nous commençons à retirer une à une les pelures de l'oignon, à déceler les couches identitaires et nous nous retrouvons, en fin de compte, face à notre mortalité et à notre vanité » (p. 22). 
Le problème, par contre, avec une telle posture, c'est qu'elle fait l'impasse sur le caractère non systématique de la démarche de Dalpé. Dans Personæ, on trouve aussi de véritables jumeaux! C'est que la frontière entre l'authentique et le fabriqué n'est pas là pour être franchie, mais littéralement habitée. Et il est impossible de déterminer chaque fois, de façon certaine, ce qu'il en est des clichés. L'identité n'est plus stable, elle devient un jeu, où la surface (ce qu'en régime d'intimité on aurait appelé le paraître) s'impose comme marqueur principal. L'identité est un effet de présence. Ce qui est bien une conséquence de l'identitéflux.

Un tel jeu sur les apparences, et sur une frontière de plus en plus poreuse entre l'authentique et le fabriqué, se retrouve aussi dans les photographies des frères Carlos et Jason Sanchez. Là encore, de singuliers effets de présence se font sentir. Une de leurs photographies, intitulée «Natural Selection» (Sanchez, 2007), montre deux chiens qui sont sur le point d'attaquer un troisième, déjà en posture de défense. Les crocs sont sortis, les poils hérissés, on croit presque entendre les grognements. La violence a été saisie sur le vif. On ne sait rien des circonstances qui ont mené à cette attaque. Le titre de la photographie nous parle de sélection naturelle, et on comprend que le chien noir fera les frais de sa différence, attaqué par deux chiens semblables dans leur pelage, noir et beige. Mais, quoi qu'il en soit de son contexte ou de sa signification, l'agression est figurée de façon non ambiguë dans cette image, d'où l'effet d’immédiateté de la représentation ressenti. 


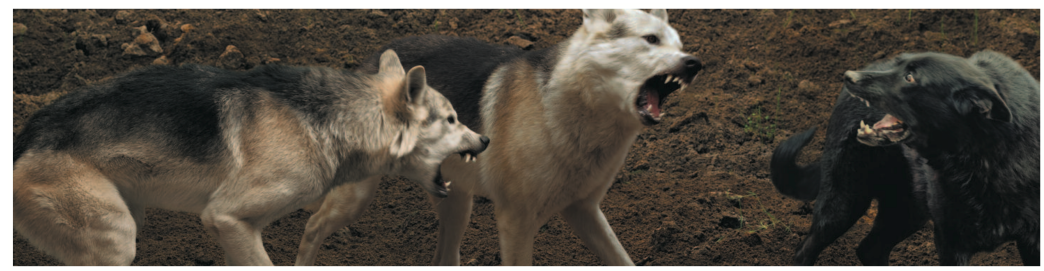

Carlos et Jason Sanchez, « Natural Selection », dans The Moment of Rupture

La charge d'agressivité est telle dans Natural Selection qu'on en oublie aisément qu'il s'agit d'une photo. Notre attention se concentre sur le contenu de l'image, plutôt que sur le médium lui-même, sur les deux chiens qui en attaquent un troisième. À aucun moment on ne pense au fait qu'il pourrait s'agir d'une agression simulée. Ce sont des chiens après tout, qui ne font pas dans la représentation et la fiction. L'agression est réelle.

Cette violence et le caractère urgent de la situation suggèrent que cette scène est spontanée. Cette photographie rend compte d'un "ça-a-été », pour reprendre l'expression de Roland Barthes (1980, p.120). L'usage du plan rapproché et l'absence de références spatiotemporelles aident à provoquer cet effet. De plus, notre peur de ce type de situation peut nous conduire à atténuer la différence entre le réel et le représenté. Lorsque nous nous sentons menacés, plus aucune différence ne tient, car cette émotion engage autre chose que notre rationalité. Elle engage notre instinct de survie, à l'image de la réaction du chien noir agressé. Or, cette scène, «croquée sur le vif », n'a rien de spontané, il s'agit d'un montage. La scène a été entièrement créée en studio à l'aide d'un éleveur de chiens. D'ailleurs, si on regarde bien les deux chiens agresseurs, on devine facilement qu'il s'agit de doubles, comme avec les photos 
de Dalpé. Il n'y a qu'un seul chien noir et beige qui attaque le noir, mais sa figure a été dédoublée par le biais du montage numérique.

Natural Selection est une pure fiction photographique, qui nous parle, comme toutes les photos des frères Sanchez, de cet espace intercalaire ouvert, en culture de l'écran marqué par une manipulation numérique des données, entre le vrai et le faux, l'authentique et le fictionnel, espace frontière qui permet à des effets de présence d'être ressentis et d'imposer leur logique symbolique. Ça nous parle. Dans le flux des données et des images, celles-ci nous disent quelque chose du monde, même si leur propos est construit de toutes pièces.

\section{Une somme d'images}

Ce qui apparaît évident, c'est que la photographie et les images, de façon plus large, sont liées à nos comportements cognitifs. On manipule les images, on les transforme, mais on s'en sert aussi pour réfléchir, pour être ému, pour se souvenir, pour assurer notre relation au monde. Les images servent à connaître et à se faire connaître. Elles sont au cœur de notre identité-flux. Réfléchir, se souvenir, c'est travailler avec des images, c'est toucher et retoucher des images. Comme le dit Gregory Chatonsky, "l'image n'est plus une représentation depuis des décennies déjà, mais un instrument. [...] C'est l'apparition d'une image-instrument, de sorte que l'écran ne se limite plus à l'inscription de l'image, il revêt également un fonctionnement instrumental et c'est pourquoi il devient tactile. Il ne concerne plus seulement le regard, mais la main. » (2008, p. 109) 
Les photos des frères Sanchez, de Pierre Dalpé et de tous ces autres photographes qui manipulent des images, s'inscrivent dans une culture de l'écran; elles prennent place dans une culture où les rapports de représentation sont déployés selon de nouveaux principes, dont la notion même d'écran permet de saisir la différence.

Nous sommes une somme d'images. Nous sommes la somme des images que nous projetons de nous et que les autres se font de nous. C'est l'un des principes de l'identité-flux. Une identité en constante négociation, tantôt passeport, tantôt monnaie d'échange.

C'est dire que la dynamique des relations en culture de l'écran n'est pas fondée sur la tradition, l'identité, la pérennité et la mémoire, marqueurs d'un sujet défini par son intimité, mais sur un autre ensemble de notions, parmi lesquelles on note la traduction, la variation, la labilité et l'oubli². Ces notions participent à la définition d'une identité-flux. Elles dessinent une expérience singulière et voient à l'apparition de modes de relations et de découverte soumis à des ajustements inédits.

\footnotetext{
${ }^{2}$ Pour une description de ces quatre termes, voir Gervais (2010).
} 


\section{Bibliographie}

AMERIKA, Mark. (2011), remixthebook, Minneapolis, University of Minnesota Press.

BARTHES, Roland. (1980), La Chambre claire. Notes sur la photographie, Paris, Cahiers du cinéma / Gallimard / Seuil

Buci-Glucksmann, Christine. (2002), La Folie du voir: Une esthétique du virtuel, Paris, Galilée.

Chatonsky, Gregory. (2008), «La répétition des limites. Diffusion, projection et immersion », dans Louise PoISSANT et Pierre Tremblay (dir.), Esthétique des arts médiatiques, Prolifération des écrans, Montréal, Presses de l'Université du Québec, p. 87-129.

DALPÉ, Pierre. (2010), «Los Hermanos Miranda », photographie parue dans Personæ, Mont St-Hilaire, Les éditions Cayenne.

GERVAIS, Bertrand et Mariève DesjARDins. (2009), « Le spectacle du corps à l'ère d'Internet. Entre virtualité et banalité », Protée, vol. 37, no 1, p. 9-23.

GERVAIS, Bertrand. (2010), "Le cyberespace: principes et esthétiques. Réflexions sur le contemporain VII», Salon double, novembre, $<$ http://salondouble.contemporain.info/antichambre/lecyberespace-principes-et-esthetiques $>$.

-. (2014), «Une figure suspendue. Reprise et dédoublement du "Falling Man" dans les fictions du 11 septembre 2001 », dans Bertrand GervaIS, Alice van der KLEI et Annie Dulong (dir.), L'Imaginaire du 11 septembre 2001. Motifs, figures et fictions, Québec, Nota bene, p. 173-193. 
MCLEOD, Dayna. (2010), Personæ, Mont Saint-Hilaire, Les éditions Cayenne.

Ricoeur, PAUl. (1990), Soi-même comme un autre, Paris, Seuil.

SANCHEZ, Carlos et Jason. (2007), The Moment of Rupture / L'instant de la rupture, Montréal, UMA, La Maison de l'image et de la photographie.

SOURKES, Cheryl. Cam Work, <http://www.cherylsourkes.com/index.htm>.

Tisseron, Serge. (1999), Comment l'esprit vient aux objets, Paris, Aubier.

—. (2001), L'Intimité surexposée, Paris, Ramsay.

—. (2006), «Les nouveaux visages de l'extimité : l'artiste et le délinquant», Esse arts + opinions aut./hiv., n ${ }^{\circ} 58$, $<$ http://esse.ca/fr/les-nouveaux-visages-de1\%E2\%80\%99extimite-l-artiste-et-le-delinquant>.

Wombell, Paul (dir.). (2013), Drone: l'image automatisée, catalogue, Mois de la photo à Montréal, Kerber, Photo Art. 


\title{
Résumé
}

L'imaginaire contemporain est marqué par une "folie du voir ", laquelle étend son emprise sur de nombreuses sphères de nos activités sémiotiques et s'inscrit comme trait saillant d'une culture de l'écran. Elle joue explicitement sur une contribution des images non seulement comme mode de représentation, mais comme modalités identitaires et processus de connaissance. C'est sur ces deux aspects, identitaire et cognitif, que s'arrête cet article, en fonction de la place qu'occupe l'image, par la voie de la photographie, et l'écran, en tant qu'interface, dans nos habitudes et nos façons de nous définir, dans la constitution d'une «identité-flux ».

\begin{abstract}
The contemporary imagination is characterized by «la folie du voir " (the madness of seeing) which extends its grip on many spheres of our semiotic activities and registers as a salient feature of screen culture. It explicitly plays on the contribution of images not only as a mode of representation but as identity terms and knowledge process as well. This article focusses on these two aspects of identity and cognitive operations, according to the role played by images (through photography) and screens (as interface) in our habits and our ways of defining ourselves, in the creation of an « identity-flow ».
\end{abstract}

